\title{
ANTHROPOMETRIC AND BIOCHEMICAL CHARACTERISTICS OF ADULT PATIENTS WITH NON-ALCOHOLIC FATTY LIVER DISEASE
}

\author{
MOUSHUMI LODH ${ }^{1}$, NAVONEEL DEBNATH ${ }^{2}$, DIPENDU MAZUMDAR ${ }^{3}$, BINIDRA BANNERJEE $^{4}$, BINITA GOSWAMI $^{5}$
}

${ }^{1}$ Department of Biochemistry, Senior Consultant, Healthworld Hospital, Durgapur, West Bengal, India. ${ }^{2}$ MBBS Student, 3rd prof, part 1, IQ City Medical College and Narayana Multispecialty Hospital, Durgapur, West Bengal, India. ${ }^{3}$ Department of Gastroenterology, Senior Consultant, HealthWorld Hospital, Durgapur, West Bengal, India. ${ }^{4}$ DNB Medicine, IQ City Medical College and Narayana Multispecialty Hospital, Durgapur, West Bengal, India. ${ }^{5}$ Department of Biochemistry, MAMC, New Delhi, Delhi, India. Email: drmoushumilodh@gmail.com

Received: 28 August 2018, Revised and Accepted: 28 September 2018

\section{ABSTRACT}

Objective: The objective of this study was to analyze the distribution of non-alcoholic fatty liver disease (NAFLD) with reference to age, gender, and socioeconomic status, to look for clinical features, anthropometric measurements, and biochemical parameters in patients of NAFLD, and to analyze statistically significant differences in biochemical parameters of lean, overweight, and obese NAFLD patients, in a medical college hospital of Durgapur.

Methods: This was a prospective, cross-sectional hospital-based study carried out over 2 months. Patients diagnosed as NAFLD based on clinical findings and ultrasonography (USG) were evaluated. Patients were classified on the basis of body mass index (BMI) and their fibrosis score calculated.

Results: The authors report several interesting findings that warrant further exploration. In this study, average age was 42 years and there was a male preponderance. $31.25 \%$ among patients who had undergone USG evaluation were diagnosed as NAFLD. Glucose, glycosylated hemoglobin, thyroidstimulating hormone (TSH), BMI, and lipid profile were significantly different in controls and cases. In NAFLD patient group, $48 \%$ were lean, $40 \%$ overweight, and $12 \%$ obese. Lean patients had significantly different alanine transaminase/aspartate transaminase, TSH, and fibrosis score compared to overweight and obese patients.

Conclusion: Authors stress on the importance of early identification of patients and stratifying them using less invasive, highly accurate, and affordable screening tools such as USG, biochemical tests, and fibrosis scores. More large-scale prospective studies can validate our observations, help physicians in early identification of patients who may benefit from therapeutic interventions, and even help them formulate more effective treatment algorithms.

Keywords: Non-alcoholic fatty liver disease, Fatty liver, Steatohepatitis, Metabolic syndrome, Fibrosis score.

(c) 2019 The Authors. Published by Innovare Academic Sciences Pvt Ltd. This is an open access article under the CC BY license (http://creativecommons. org/licenses/by/4. 0/) DOI: http://dx.doi.org/10.22159/ajpcr.2019.v12i1.29278

\section{INTRODUCTION}

Non-alcoholic fatty liver disease (NAFLD) is the most common cause of liver disease in the Western world [1]. The spectrum of abnormalities includes a simple accumulation of triglycerides (TG) in the hepatocytes (hepatic steatosis), hepatic steatosis with inflammation (steatohepatitis), fibrosis, and cirrhosis. The prevalence of NAFLD is around $9-32 \%$ in the general Indian population [2]. It is present in up to $90 \%$ of obese patients [3]. NAFLD is also the most common cause of elevated liver enzymes [4]. It can progress to hepatitis and hepatocarcinoma. Understanding the epidemiology and possible correlations of dyslipidemia, glycemic status and other risk factors with NAFLD are essential for developing effective treatment and prevention strategies, especially for people of this part of eastern India.

Hence, this study was undertaken to evaluate the risk factors and biochemical characteristics of NAFLD with the aim to analyze the distribution of NAFLD with reference to age, gender, and socioeconomic status, to look for clinical features, anthropometric measurements, and biochemical parameters in patients of NAFLD, and to analyze statistically significant differences in biochemical parameters of patients and controls. Such a study has not been documented in this part of West Bengal.

\section{METHODS}

This was a prospective, cross-sectional hospital-based study carried out over 2 months. 160 patients had undergone ultrasonographic examination of the liver from medicine and gastroenterology outpatient department (OPD) of a tertiary care teaching hospital. 50 patients diagnosed as NAFLD by ultrasonography (USG) criteria were taken up for study. Patients were evaluated on history, clinical examination, anthropometric measurements, biochemical test, and USG of the abdomen. 30 age- and sex-matched healthy subjects from staff and students of medical college and hospital were taken as control group.

In this study, out of about 375 patients who presented to gastroenterology OPD for various complaints, 160 patients with elevated liver enzymes had undergone ultrasonographic study of liver, of which 50 patients had been diagnosed as NAFLD. Following patients were included in the study: NAFLD patients diagnosed with USG, patient without a history of alcohol consumption of more than $30 \mathrm{~g} /$ day for men and $20 \mathrm{~g} /$ day for women, and no other existing causes of chronic liver disease. Subjects with any of the following were excluded: Positive serum level for hepatitis B surface antigen (using enzyme-linked immunosorbent assay [ELISA], Enzygnost, Siemens); positive serum level for hepatitis $C$ virus antibody(using ELISA), alcohol use more than $20 \mathrm{~g} /$ day; and active malignancy, history of chronic liver diseases including Wilson's disease, hemochromatosis, and autoimmune hepatitis, and history of use of drugs causing liver function abnormality (hepatotoxic drugs such as tamoxifen, amiodarone, and methotrexate).

Consent for including data for the purpose of the study was obtained from each patient at the time of enrolment. The study protocol was approved by the Institutional Ethics Committee. Patient selection criteria were based on Dowman et al. [1].

Monthly income as indicator of socioeconomic status was recorded as follows: Upper socioeconomic status - 30,000/- and above per month, 
middle socioeconomic status - 10,000-30,000/- per month, and lower socioeconomic status - $<10,000 /$ - per month. Background was recorded as urban or rural.

Weight, height, waist circumference (WC), and blood pressure (BP) including systolic BP (SBP) and diastolic BP (DBP) were measured. Body mass index (BMI) was calculated according to the World Health Organization (WHO) [5]. Lean patients had BMI 18.5-24.9 kg/m², overweight patients had BMI $25-29.9 \mathrm{~kg} / \mathrm{m}^{2}$, and obese had a BMI $>30 \mathrm{~kg} / \mathrm{m}^{2}$.

Waistline was measured at midpoint between lower chest wall and iliac crest with the patient standing. WC $>90 \mathrm{~cm}$ in male and $>80 \mathrm{~cm}$ in female would indicate central obesity.

NAFLD was diagnosed by USG of liver using the standardized criteria of increased echogenicity of liver texture compared to the right kidney, lack of transmission of sound to the posterior diaphragmatic interface, lack of visibility of vascular structure due to ill-defined portal walls invaded by fat, and increased liver size as measured in midclavicular line [6]. Fatty liver was diagnosed in the presence of two of the three observations of bright hepatic echotexture compared to kidney and spleen, blurring of hepatic veins, and loss of deep echodiscontinuous diaphragm.

All respondents donated blood samples after over $10 \mathrm{~h}$ of overnight fasting for biochemical measurement including fasting blood glucose, total cholesterol (TC), high-density lipoprotein cholesterol (HDL-C), low-density lipoprotein cholesterol (LDL-C), TG, aspartate aminotransferase (AST), alanine aminotransferase (ALT), $\gamma$-glutamyl transferase, alkaline phosphatase, and C-reactive protein (Siemens Dimension $\mathrm{R}_{\mathrm{x}} \mathrm{L}$ Max Biochemistry Analyzer). Other tests done were complete blood count (Sysmex KX 21), prothrombin time (Sysmex CA50 Analyzer), serology for antinuclear antibody, markers for viral hepatitis A, B, C, and E by ELISA, serum ferritin, ceruloplasmin, thyroidstimulating hormone (TSH) (chemiluminescence, immunoassay on Siemens Advia Centaur XP), and glycosylated hemoglobin $\left(\mathrm{HbA}_{1} \mathrm{C}\right)$ by HPLC (Transasia Hb Vario).

We have used NAFLD fibrosis score (www.nafld score.com) which is a validating scoring system comprising of six parameters (age, hyperglycemia, BMI, platelet count, albumin, and AST/ALT ratio). This helps to classify patients into groups with low, intermediate, and high probability/risk for liver-related complications [7].

All values are reported as the mean and standard deviation for continuous variables and the number (percent) for categoric variables. Comparison of groups was performed using analysis of variance and Fisher tests. Statistical significance was set at $p<0.05$. All statistical calculations were performed using Microsoft Excel.

\section{RESULTS AND DISCUSSION}

With the increased incidence of diabetes mellitus, obesity, and insulin resistance in India in the past two decades, it is only logical to expect an increase in the incidence of NAFLD in India. Several diagnostic modalities have been used for the diagnosis of fatty liver, including ultrasound, liver enzymes, computed tomography, magnetic resonance imaging spectroscopy (MRS), and liver biopsy. Ultrasound was the preferred method for our study due to the relatively low cost, fair accuracy, and noninvasive nature. Liver enzymes may not be elevated in NAFLD patients. Liver biopsy through the gold standard is not feasible in all suspected cases. The accuracy of MRS is overshadowed by its huge cost.

In this study, $31.25 \%$ of 160 patients who had undergone USG evaluation were diagnosed as NAFLD. About two-third (62\%) patients were from rural areas. $62 \%$ of NAFLD patients were middle socioeconomic class. Our study shows that weight and BMI were significantly higher in cases compared to controls $(p<0.05)$. Mean age of the patients is 42 years. More males were affected as has been depicted in Table 1 .
We classified cases and controls into lean, overweight, and obese with BMI ranging $18.5-25 \mathrm{~kg} / \mathrm{m}^{2}$ for lean, $25-30 \mathrm{~kg} / \mathrm{m}^{2}$ for overweight, and $>30 \mathrm{~kg} / \mathrm{m}^{2}$ for obese [5]. In the control group, $77 \%$ were lean, $20 \%$ overweight, and $3 \%$ obese. In NAFLD patient group, $48 \%$ were lean, $40 \%$ overweight, and $12 \%$ obese. Lean and overweight together constituted $88 \%$ of the total patient population.

Our study shows that lipid profile was significantly higher in cases compared to controls. Mean TSH is significantly higher among cases when compared with that of control group. Mean serum glucose level is significantly higher among cases. All these biochemical findings are summarized in Table 2 .

Mean total serum cholesterol, triglyceride, and LDL cholesterol level of control group were significantly lower than patient group. A study [8] showed significant association of elevated total serum cholesterol and NAFLD as when compared with controls. However, other authors $[9,10]$ showed no significant association of total serum cholesterol and NAFLD. Singh et al. [11] found that serum triglyceride level was significantly higher in NAFLD group when compared to controls. Nigam et al. [12] showed significant association of serum triglyceride level and NAFLD. Mean serum HDL level is low among NAFLD patients when compared with control group, and this is statistically significant $(\mathrm{p}<0.005)$. Odds ratio as per another study [13] of low HDL-C $(<40 \mathrm{mg} / \mathrm{dl}$ in men or $<50 \mathrm{mg} / \mathrm{dl}$ in women) is $1.61-2.0$. Although most patients of NAFLD are obese or overweight, lean NAFLD may represent a different pathophysiology, rather than merely a by-product of different BMI [14]. Our lean patients' lipid profile did not differ significantly from overweight and obese patients.

As per a study [15], the entire histologic spectrum of NAFLD can be seen in subjects with normal ALT. Our study, like others' $[12,16]$, showed a significant association of ALT with NAFLD. However, the study of Gupte et al. [17] showed no significant association between the two.

Table 1: Anthropometric measurement of cases and controls $($ mean \pm SD)

\begin{tabular}{llll}
\hline Parameter & Control $(\mathbf{n = 3 0})$ & Case $(\mathbf{n = 5 0})$ & $\mathbf{p}$ value \\
\hline Age & $31.2 \pm 8.8$ & $42 \pm 12$ & NS \\
Sex $(\mathrm{M} / \mathrm{F})$ & $21 / 9$ & $30 / 20$ & \\
Height $(\mathrm{m})$ & $1.7 \pm 0.1$ & $1.7 \pm 0.1$ & $\mathrm{NS}$ \\
Weight $(\mathrm{kg})$ & $67.8 \pm 10.5$ & $70.5 \pm 15.4$ & $<0.05$ \\
BMI $\left(\mathrm{Kg} / \mathrm{m}^{2}\right)$ & $23.6 \pm 2.9$ & $25.6 \pm 4$ & $<0.05$ \\
Waist/hip ratio & $0.9 \pm 0.1$ & $0.9 \pm 0.3$ & NS \\
\hline
\end{tabular}

NS: Not significant, BMI: Body mass index

Table 2: Biochemical characteristics in cases and controls (Mean \pm SD)

\begin{tabular}{llll}
\hline Parameter & Control $(\mathbf{n = 3 0})$ & Case $(\mathbf{n = 5 0})$ & p value \\
\hline Cholesterol (mg/dl) & $151.4 \pm 18$ & $181.2 \pm 46$ & $\mathrm{p}<0.001$ \\
Triglyceride (mg/dl) & $115 \pm 17.4$ & $147.4 \pm 56.7$ & $\mathrm{p}<0.005$ \\
HDL cholesterol (mg/dl) & $49.9 \pm 7.7$ & $43.3 \pm 10.5$ & $\mathrm{p}<0.005$ \\
LDL cholesterol (mg/dl) & $78.7 \pm 10.1$ & $98.1 \pm 30.9$ & $\mathrm{p}<0.001$ \\
AST (U/L) & $21.8 \pm 8$ & $46.6 \pm 22.7$ & $\mathrm{NS}$ \\
ALT (u/l) & $30 \pm 8.2$ & $87.7 \pm 51.5$ & $\mathrm{p}<0.05$ \\
Albumin (g/dl) & $4.3 \pm 0.2$ & $4.3 \pm 0.5$ & $\mathrm{NS}$ \\
TSH (mIU/L) & $2.3 \pm 1.2$ & $3.6 \pm 1.9$ & $\mathrm{p}<0.001$ \\
Fasting glucose (mg/dl) & $102 \pm 5.7$ & $115.2 \pm 45$ & $\mathrm{p}=0.001$ \\
Ferritin (ng/ml) & $117.6 \pm 87.3$ & $146.7 \pm 97.3$ & $\mathrm{NS}$ \\
Ceruloplasmin $(\mathrm{mg} / \mathrm{dl})$ & $26 \pm 4.2$ & $30.4 \pm 6.4$ & $\mathrm{NS}$ \\
HBA C $(\%)$ & $4.9 \pm 0.5$ & $5.0 \pm 0.9$ & $\mathrm{p}=0.001$ \\
Platelet $\left(10^{3} / \mathrm{mm}^{3}\right)$ & $2.9 \pm 0.7$ & $3.1 \pm 1$ & $\mathrm{NS}$ \\
\hline
\end{tabular}

HBA C: Hemoglobin, TSH: Thyroid-stimulating hormone, ALT: Alanine aminotransferase, AST: Aspartate aminotransferase, LDL: Low-density lipoprotein, HDL: High-density lipoprotein 
Plasma glucose level and HBA1C were higher among NAFLD cases when compared with control group, as were observed by other authors [16,18-21]. Mean HBA $\mathrm{C}_{1}$ level in our study was $4.9 \pm 0.5$ among control group and $4.8 \pm 0.9$ among NAFLD cases. When we divided the patient group into lean, overweight, and obese as per the WHO classification, we found that HDL-C was significantly different in obese compared to lean. AST/ALT ratios were significantly different on comparing lean with overweight and lean with obese.

In a recent study [22], it was observed that BMI, SBP, DBP, TC, HDL cholesterol, LDL cholesterol, and triglyceride were significantly different in lean patients with NAFLD compared to control group. Furthermore, WC in men and hip circumference in women were higher in lean patients with NAFLD. Feng et al. [23] noted that total free fatty acid (FFA) profiles were insignificantly different between lean $(2093.33 \pm 558.11 \mu \mathrm{g} / \mathrm{ml})$ and overweight $(2420.81 \pm 555.18 \mu \mathrm{g} / \mathrm{ml})$ NAFLD patients, obese NAFLD $(2739.01 \pm 810.35 \mu \mathrm{g} / \mathrm{ml})$ presented most significantly elevated $(\mathrm{p}<0.05)$ total FFA profiles.

Obesity is known to be a good predictor of hepatic steatosis and disease progression [24]. Steatosis is at least 2 times more frequent in overweight than in lean subjects. The lipids which are elevated inside the hepatocytes may be TG, FFA, diacylglycerol, cholesterol, cholesterol esters, phospholipids, and ceramides. In a study in India [25], it was reported that a statistically significant difference between the BMI, waist-hip ratio, mean triglyceride, and AST and ALT values exists between the obese and non-obese patients of NAFLD.

In Table 3, the mean values of various parameters in 24 lean, 20 overweight, and 6 obese patients are shown. Weight difference between overweight and obese was statistically significant. HDL cholesterol levels were significantly different in obese and lean patients. AST/ALT ratio was statistically significant on comparing lean with obese and again on comparing lean with overweight. TSH was significantly different in lean compared to overweight and also lean compared to obese.

The adipose tissues of lean NAFLD patients may have more proinflammatory potential. In lean NAFLD patients, visceral adipocytes' cytokine secretion and small intestinal bacterial overgrowth and the resulting endotoxemia have been shown to be the culprits [14].

TSH levels showed statistically significant difference when we compared lean with overweight and lean with obese patients. The thyroid gland is significantly involved in energy homeostasis, lipid and carbohydrate metabolism, and adipogenesis. In a clinical setting, subclinical hypothyroidism has been associated with metabolic syndrome, cardiovascular mortality, and disturbance of lipid metabolism. The prevalence of hypothyroidism was reported to a range from $15.2 \%$ to $36.3 \%$ [26] among patients with NAFLD or non-alcoholic steatohepatitis.

To clarify the mechanism of thyroid dysfunction and NAFLD, studies mention elevated leptin and visfatin, both adipocytokines seen in NAFLD [27]. Leptin is involved in the regulation of appetite. Elevated levels are seen in obesity. They induce collagen synthesis in the liver and stimulate hepatic insulin resistance [28]. Patients with NAFLD have abnormal lipid profiles. Thyroid hormones induce their effects on lipid metabolism through thyroid hormone receptor $\beta$, which is expressed in liver [29]. Thyroid hormone receptor activation results in a reduction in body weight and fat as well as a decrease in cholesterol and triglyceride levels, which takes place only in hepatocytes [30,31]. Stress also elevates leptin levels and can be an indirect cause for NAFLD. While one study [32] advocates the use of Ezenus in the treatment of stress, another study [33] mentions that flavonoids present in Vitis Vinifera seeds have powerful antioxidant and anti-inflammatory properties, maintain hepatocytes structure integrity, and decrease ALT levels in NAFLD.

In our study, we also used the fibrosis score as a risk assessment for NAFLD patients developing complications of fibrosis, which has been depicted in Fig. 1.

In Fig. 1, we found that $70 \%$ of patients had NAFLD score $<1.5$. They are at low probability of progressing to liver fibrosis. $10 \%$ of NAFLD patients had a score $>0.67$ and were at high probability of progressing to fibrosis while $20 \%$ of patients with score ranging from $>1.5$ to $<0.67$ are at intermediate probability of progressing to liver fibrosis [7]. Compared to lean patients, overweight and obese showed significantly higher fibrosis score $(\mathrm{p}<0.05)$.

Table 3: Comparison of anthropometric and biochemical parameters between lean overweight and obese patients

\begin{tabular}{|c|c|c|c|c|c|c|}
\hline Parameter & Lean [1] & Overweight [2] & Obese [3] & $p$ value between 1 and 2 & p value between 2 and 3 & $p$ value between 1 and 3 \\
\hline Age (years) & $40 \pm 11.4$ & $45.3 \pm 11.8$ & $35.8 \pm 11.5$ & NS & NS & NS \\
\hline Sex (M:F) & $13: 11$ & $12: 08$ & 04:02 & NS & NS & NS \\
\hline Height (m) & $1.6 \pm 0.1$ & $1.7 \pm 0.1$ & $1.7 \pm 0.1$ & NS & NS & NS \\
\hline Weight (kg) & $60.6 \pm 7.5$ & $75.8 \pm 9.2$ & $92.4 \pm 24.2$ & NS & $\mathrm{p}<0.05$ & NS \\
\hline BMI $\left(\mathrm{kg} / \mathrm{m}^{2}\right)$ & $22.3 \pm 1.4$ & $27.3 \pm 1.4$ & $33.1 \pm 3.2$ & NS & NS & NS \\
\hline Waist/hip ratio & $0.9 \pm 0.2$ & $0.9 \pm 0.4$ & $0.8 \pm 0.5$ & NS & NS & NS \\
\hline Cholesterol (mg/dl) & $171.1 \pm 40.6$ & $194.9 \pm 53.5$ & $176.5 \pm 31.6$ & NS & NS & NS \\
\hline Triglyceride (mg/dl) & $141.7 \pm 55.4$ & $151.4 \pm 63.2$ & $157.3 \pm 43.3$ & NS & NS & NS \\
\hline HDL cholesterol (mg/dl) & $41 \pm 8.8$ & $43.7 \pm 11.6$ & $51.3 \pm 10.7$ & NS & NS & $\mathrm{p}<0.05$ \\
\hline LDL cholesterol (mg/dl) & $93.1 \pm 29.6$ & $105.1 \pm 33$ & $94.7 \pm 29$ & NS & NS & NS \\
\hline AST (U/L) & $49.7 \pm 22.3$ & $38.1 \pm 15.2$ & $62.5 \pm 35.6$ & NS & $\mathrm{p}<0.05$ & NS \\
\hline ALT (U/L) & $103.6 \pm 56.4$ & $67.5 \pm 41.5$ & $91.5 \pm 44.3$ & NS & NS & NS \\
\hline AST/ALT & $0.5 \pm 0.2$ & $0.7 \pm 0.3$ & 0.70 .2 & $\mathrm{p}<0.05$ & NS & $\mathrm{p}<0.05$ \\
\hline Albumin (g/dl) & $4.2 \pm 0.5$ & $4.3 \pm 0.5$ & $4.4 \pm 0.4$ & NS & NS & NS \\
\hline TSH (mIU/L) & $3 \pm 1.2$ & $4.1 \pm 2.4$ & $4.2 \pm 2.5$ & $\mathrm{p}<0.05$ & NS & $\mathrm{p}<0.05$ \\
\hline Glucose (mg/dl) & $120.3 \pm 54.1$ & $116.3 \pm 30.9$ & $114.3 \pm 46$ & NS & NS & NS \\
\hline Ferritin (ng/mL) & $132.6 \pm 59.9$ & $175.8 \pm 123.5$ & $161.4 \pm 113.1$ & NS & NS & NS \\
\hline Ceruloplasmin (mg/dL) & $30.5 \pm 6.7$ & $29.7 \pm 6.6$ & $32.5 \pm 5.5$ & NS & NS & NS \\
\hline $\mathrm{HBA}_{1} \mathrm{C}(\%)$ & $4.7 \pm 1$ & $5.2 \pm 1.1$ & $5.6 \pm 0.8$ & NS & NS & NS \\
\hline Platelet $\left(10^{3} / \mathrm{mm}^{3}\right)$ & $3 \pm 0.9$ & $3.2 \pm 1$ & $3.3 \pm 0.8$ & NS & NS & NS \\
\hline
\end{tabular}

HBA C: Hemoglobin, TSH: Thyroid-stimulating hormone, ALT: Alanine aminotransferase, AST: Aspartate aminotransferase, LDL: Low-density lipoprotein,

HDL: High density lipoprotein, NS: Not significant 


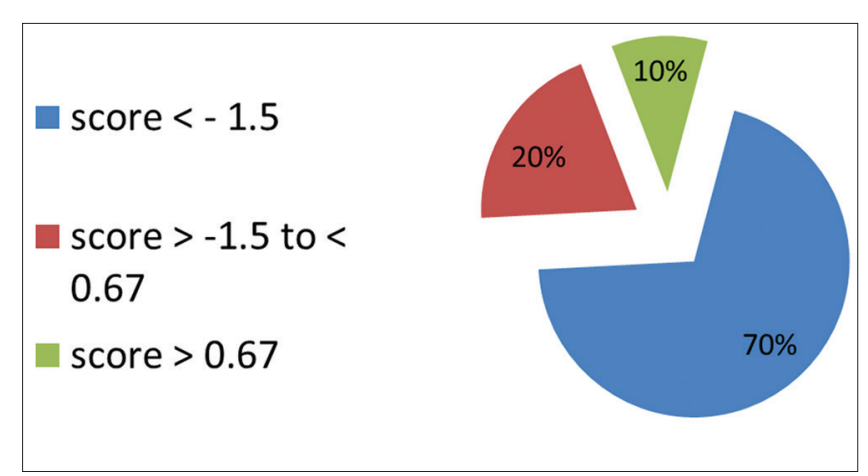

Fig. 1: Non-alcoholic fatty liver disease fibrosis score as an indicator of the probability of fibrosis in patients

\section{CONCLUSION}

Obesity, insulin resistance, and metabolic syndrome prevalence are continuously increasing. This has grave implications because the resultant increase in NAFLD patients will translate into increased numbers of patients with complications such as cirrhosis, liver failure, and hepatocellular carcinoma. Here lies the importance of early identification of the disease using less invasive,highly accurate and affordable screening tools, like USG and biochemical tests.More largescale prospective studies can validate our observations, help physicians in early identification of patients who may benefit from therapeutic interventions, and even help them formulate more effective treatment algorithms.

\section{ACKNOWLEDGMENT}

This was done under ICMR STS Project for MBBS students.

\section{AUTHORS' CONTRIBUTIONS}

ML designed the study, wrote the protocol, and wrote the first draft of study; ND collected data and did data analysis; DM helped in patient selection and diagnosis, assisted with data collection. BB helped in patient selection and diagnosis, assisted with data collection. BG performed the literature searches and wrote the final draft. All authors read, provided critical inputs, and approved the final manuscript.

\section{CONFLICTS OF INTEREST}

The authors declared that they have no conflicts of interest.

\section{REFERENCES}

1. Dowman JK, Tomlinson JW, Newsome PN. Systematic review: The diagnosis and staging of non-alcoholic fatty liver disease and nonalcoholic steatohepatitis. Aliment Pharmacol Ther 2011;33:525-40.

2. Kalra S, Vithalani M, Gulati G, Kulkarni CM, Kadam Y, Pallivathukkal J, et al. Study of prevalence of nonalcoholic fatty liver disease (NAFLD) in type 2 diabetes patients in India (SPRINT). J Assoc Physicians India 2013;61:448-53.

3. Barros F, Setúbal S, Martinho JM, Ferraz L, Gaudêncio A. Correlation of non-alcoholic fatty liver disease and features of metabolic syndrome in morbidly obese patients in the preoperative assessment for bariatric surgery. Arq Bras Cir Dig 2016;29:260-3.

4. Vernon G, Baranova A, Younossi ZM. Systematic review: The epidemiology and natural history of non-alcoholic fatty liver disease and non-alcoholic steatohepatitis in adults. Aliment Pharmacol Ther 2011;34:274-85.

5. World Health Organization. Obesity: Preventing and Managing the Global Epidemic Report of a WHO Consultation (WHO Technical Report Series 894); 2000. p. 9.

6. Farrell GC, Chitturi S, Lau GK, Sollano JD, Asia-Pacific Working Party on NAFLD. Guidelines for the assessment and management of non-alcoholic fatty liver disease in the Asia-pacific region: Executive summary. J Gastroenterol Hepatol 2007;22:775-7.

7. Treeprasertsuk S, Björnsson E, Enders F, Suwanwalaikorn S,
Lindor KD. NAFLD fibrosis score: A prognostic predictor for mortality and liver complications among NAFLD patients. World J Gastroenterol 2013;19:1219-29.

8. Mahaling DU, Basavaraj MM, Bika AJ. Comparison of lipid profile in different grades of non-alcoholic fatty liver disease diagnosed on ultrasound. Asian Pac J Trop Biomed 2013;3:907-12.

9. Abangah G, Yousefi A, Asadollahi R, Veisani Y, Rahimifar P, Alizadeh S, et al. Correlation of body mass index and serum parameters with ultrasonographic grade of fatty change in non-alcoholic fatty liver disease. Iran Red Crescent Med J 2014;16:e12669.

10. Agarwal R, Mishra S, Dixit VK, Rai S. Non alcoholic fatty liver disease and metabolic syndrome. Indian J Prev Soc Med 2011;42:2-3.

11. Singh SP, Singh A, Misra D, Misra B, Pati GK, Panigrahi MK, et al. Risk factors associated with non-alcoholic fatty liver disease in Indians: A case-control study. J Clin Exp Hepatol 2015;5:295-302.

12. Nigam P, Bhatt SP, Misra A, Vaidya M, Dasgupta J, Chadha DS, et al. Non-alcoholic fatty liver disease is closely associated with sub-clinical inflammation: A case-control study on Asian Indians in north India. PLoS One 2013;8:e49286.

13. Mohan V, Farooq S, Deepa M, Ravikumar R, Pitchumoni CS. Prevalence of non-alcoholic fatty liver disease in urban south Indians in relation to different grades of glucose intolerance and metabolic syndrome. Diabetes Res Clin Pract 2009;84:84-91.

14. Kumar R, Mohan S. Non-alcoholic fatty liver disease in lean subjects: Characteristics and implications. J Clin Transl Hepatol 2017;5:216-23.

15. Mofrad P, Contos MJ, Haque M, Sargeant C, Fisher RA, Luketic VA, et al. Clinical and histologic spectrum of nonalcoholic fatty liver disease associated with normal ALT values. Hepatology 2003;37:1286-92.

16. Ghamar-Chehreh ME, Khedmat H, Amini M, Taheri S. Predictive factors for ultrasonographic grading of nonalcoholic fatty liver disease. Hepat Mon 2012;12:e6860.

17. Gupte P, Amarapurkar D, Agal S, Baijal R, Kulshrestha P, Pramanik S, et al. Non-alcoholic steatohepatitis in Type 2 diabetes mellitus. J Gastroenterol Hepatol 2004;19:854-8.

18. Bellentani S, Tiribelli C, Saccoccio G, Sodde M, Fratti N, De Martin C, et al. Prevalence of chronic liver disease in the general population of northern Italy: The dionysos study. Hepatology 1994;20:1442-9.

19. Yano E, Tagawa K, Yamaoka K, Mori M. Test validity of periodic liver function tests in a population of Japanese male bank employees. J Clin Epidemiol 2001;54:945-51.

20. Su CC, Wang K, Hsia TL, Chen CS, Tung TH. Association of nonalcoholic fatty liver disease with abnormal aminotransferase and postprandial hyperglycemia. J Clin Gastroenterol 2006;40:551-4.

21. Ma H, Xu C, Xu L, Yu C, Miao M, Li Y, et al. Independent association of hbA1c and nonalcoholic fatty liver disease in an elderly Chinese population. BMC Gastroenterol 2013;13:3.

22. Naderian M, Kolahdoozan S, Sharifi AS, Garmaroudi G, Yaseri M, Poustchi $\mathrm{H}$, et al. Assessment of lean patients with non-alcoholic fatty liver disease in a middle income country; prevalence and its association with metabolic disorders: A cross-sectional study. Arch Iran Med 2017;20:211-7.

23. Feng R, Luo C, Li C, Du S, Okekunle AP, Li Y, et al. Free fatty acids profile among lean, overweight and obese non-alcoholic fatty liver disease patients: A case control study. Lipids Health Dis 2017;16:165.

24. Machado MV, Cortez-Pinto H. Non-alcoholic fatty liver disease: What the clinician needs to know. World J Gastroenterol 2014;20:12956-80.

25. Patell R, Dosi R, Joshi H, Sheth S, Shah P, Jasdanwala S, et al. Nonalcoholic fatty liver disease (NAFLD) in obesity. J Clin Diagn Res 2014;8:62-6.

26. Eshraghian A, Hamidian Jahromi A. Non-alcoholic fatty liver disease and thyroid dysfunction: A systematic review. World J Gastroenterol 2014;20:8102-9.

27. Chung GE, Kim D, Kim W, Yim JY, Park MJ, Kim YJ, et al. Nonalcoholic fatty liver disease across the spectrum of hypothyroidism. J Hepatol 2012;57:150-6.

28. Oswal A, Yeo G. Leptin and the control of body weight: A review of its diverse central targets, signaling mechanisms, and role in the pathogenesis of obesity. Obesity (Silver Spring) 2010;18:221-9.

29. Hulbert AJ. Thyroid hormones and their effects: A new perspective. Biol Rev Camb Philos Soc 2000;75:519-631.

30. Grover GJ, Mellström K, Ye L, Malm J, Li YL, Bladh LG, et al. Selective thyroid hormone receptor-beta activation: A strategy for reduction of weight, cholesterol, and lipoprotein (a) with reduced cardiovascular liability. Proc Natl Acad Sci U S A 2003;100:10067-72.

31. Erion MD, Cable EE, Ito BR, Jiang H, Fujitaki JM, Finn PD, et al. Targeting thyroid hormone receptor-beta agonists to the liver reduces 
cholesterol and triglycerides and improves the therapeutic index. Proc Natl Acad Sci U S A 2007;104:15490-5.

32. Khandelwal VK, Singh P, Ravingerova T, Chaudhary M. Evaluation of Ezenus in an experimental model of diet-induced alcoholic and non-alcoholic fatty liver condition in rats. Int J Pharm Pharm Sci
2016;7:247-52.

33. Ahmed HH, Salem AM, Mohamed MR, Shahat AA, Khalil WK, Mohamed SH. Experimental evidences for the promising therapeutic role of Vitis vinifera seed extract against nonalcoholic steatohepatitis. Int J Pharm Pharm Sci 2015:7:417-24. 